\title{
Velocity-space tomography using prior information at MAST
}

\author{
Madsen, B.; Salewski, Mirko; Huang, J.; Jacobsen, A.S.; Jones, O.; McClements, K. G.
}

\section{Published in:}

Review of Scientific Instruments

Link to article, DOI:

$10.1063 / 1.5035498$

Publication date:

2018

Document Version

Publisher's PDF, also known as Version of record

Link back to DTU Orbit

Citation (APA):

Madsen, B., Salewski, M., Huang, J., Jacobsen, A. S., Jones, O., \& McClements, K. G. (2018). Velocity-space tomography using prior information at MAST. Review of Scientific Instruments, 89(10), [10D125]. https://doi.org/10.1063/1.5035498

\section{General rights}

Copyright and moral rights for the publications made accessible in the public portal are retained by the authors and/or other copyright owners and it is a condition of accessing publications that users recognise and abide by the legal requirements associated with these rights.

- Users may download and print one copy of any publication from the public portal for the purpose of private study or research.

- You may not further distribute the material or use it for any profit-making activity or commercial gain

- You may freely distribute the URL identifying the publication in the public portal 


\section{Velocity-space tomography using prior information at MAST}

B. Madsen, M. Salewski, J. Huang, A. S. Jacobsen, O. Jones, K. G. McClements, and MAST Team

Citation: Review of Scientific Instruments 89, 10D125 (2018); doi: 10.1063/1.5035498

View online: https://doi.org/10.1063/1.5035498

View Table of Contents: http://aip.scitation.org/toc/rsi/89/10

Published by the American Institute of Physics

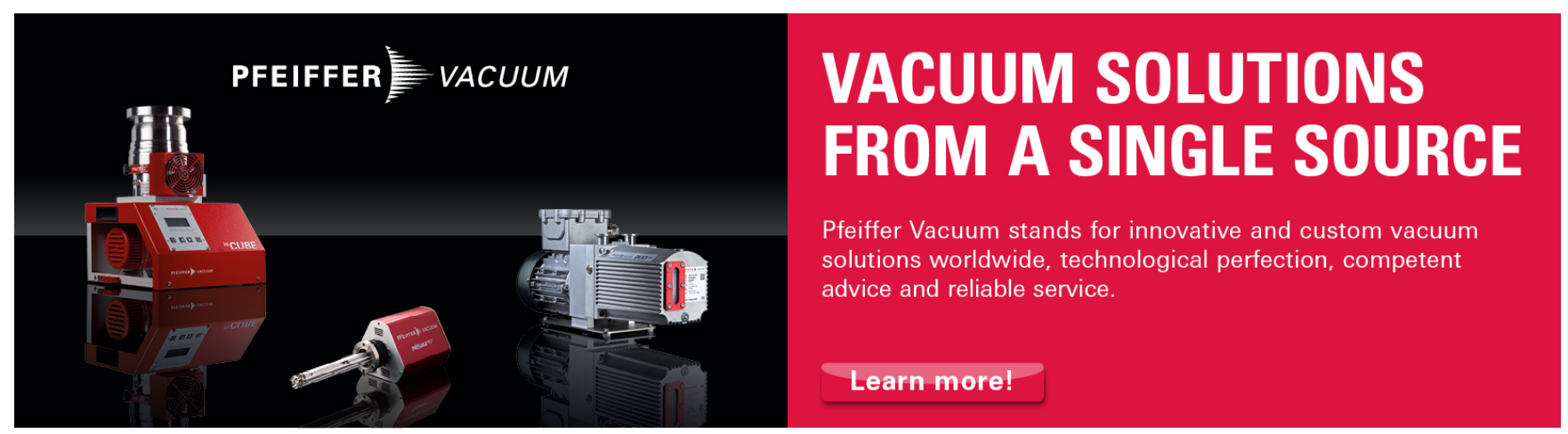




\title{
Velocity-space tomography using prior information at MAST
}

\author{
B. Madsen, ${ }^{1, \text { a) }}$ M. Salewski, ${ }^{1}$ J. Huang, ${ }^{2}$ A. S. Jacobsen, ${ }^{3}$ O. Jones, ${ }^{4}$ K. G. McClements, ${ }^{4}$ \\ and MAST Team ${ }^{4, b)}$ \\ ${ }^{1}$ Department of Physics, Technical University of Denmark, Kgs. Lyngby, Denmark \\ ${ }^{2}$ Institute of Plasma Physics, Chinese Academy of Sciences, P.O. 1126, 230031 Hefei, Anhui, China \\ ${ }^{3}$ Max-Planck-Institut für Plasmaphysik, Garching, Germany \\ ${ }^{4}$ CCFE, Culham Science Centre, Abingdon, Oxfordshire OX14 3DB, United Kingdom
}

(Presented 19 April 2018; received 16 April 2018; accepted 18 June 2018; published online 15 October 2018)

\begin{abstract}
Velocity-space tomography provides a way of diagnosing fast ions in a fusion plasma by combining measurements from multiple instruments. We use a toroidally viewing and a vertically viewing fastion D-alpha diagnostic installed on the mega-amp spherical tokamak (before the upgrade) to perform velocity-space tomography of the fast-ion distribution function. To make up for the scarce amount of data, prior information is included in the inversions. We impose a non-negativity constraint, suppress the distribution in the velocity-space region associated with null-measurements, and encode the belief that the distribution function does not extend to energies significantly higher than those expected neoclassically. This allows us to study the fast-ion velocity distributions and the derived fast-ion densities before and after a sawtooth crash. https://doi.org/10.1063/1.5035498
\end{abstract}

\section{INTRODUCTION}

It is crucial to study the behavior and confinement of energetic ions since they are essential to heat the plasma and, in fusion reactors, might damage the first wall if they escape the plasma. ${ }^{1}$ In deuterium plasmas, one method of doing so is by fast-ion D-alpha (FIDA) spectroscopy ${ }^{2,3}$ which has been implemented in numerous tokamaks and stellarators around the world. ${ }^{3-9}$ FIDA measures the Balmer-alpha radiation from neutralized fast ions following charge-exchange reactions. The neutrals donating the electrons are mostly those injected into the plasma by neutral beam injection (NBI). The spatial measurement volumes of the FIDA views are thus defined by the overlap between the lines-of-sight and the neutral beam. In the mega-amp spherical tokamak (MAST), toroidally and vertically directed FIDA views were installed. ${ }^{9}{ }^{9} 10$ We combine measurements from this dual-view FIDA system on MAST in reconstructions of the fast-ion distribution functions before and after a sawtooth crash providing essential information about the resulting fast-ion density decrease. Similar investigations have been carried out at ASDEX Upgrade., ${ }^{71-13}$ Sawtooth crashes also redistribute alpha-particles ${ }^{14}$ which is important with respect to ITER.

The tomography problem based on FIDA measurements at MAST is a scarce-data problem, and parts of velocityspace are covered by only one view. Therefore, tomographic inversion methods ${ }^{13}$ used for reconstructions of the fast-ion velocity distribution from FIDA measurements at ASDEX Upgrade are plagued by severe artifacts here. To make up for the scarce amount of data, we include the outer shape of the

Note: Paper published as part of the Proceedings of the 22nd Topical Conference on High-Temperature Plasma Diagnostics, San Diego, California, April 2018.

a) birma@fysik.dtu.dk

b) See the authors list in I. T. Chapman et al., Nucl. Fusion 55, 104008 (2015). neoclassically expected distribution as a new type of prior information in the tomographic inversions. This enables the reconstructions of the fast-ion velocity distributions before and after a sawtooth crash.

The paper is organized as follows. Section II introduces the modelled and experimental signals. The inversion methods are described in Sec. III, whilst Sec. IV illustrates the methods on synthetic data. This is used to reconstruct the fast-ion velocity distributions before and after a sawtooth crash in Sec. V. Section VI concludes the paper.

\section{MODELLED AND EXPERIMENTAL SIGNALS}

MAST $^{15}$ is a low aspect ratio $R / a \approx 1.3$ tokamak with major radius $R \approx 0.85 \mathrm{~m}$ and minor radius $a \approx 0.65 \mathrm{~m}$. We study the fast-ion distributions before $(t=0.253 \mathrm{~s})$ and after $(t=0.279 \mathrm{~s})$ a sawtooth crash observed by the dual-view FIDA diagnostics with lines-of-sight intersecting the NBI beam $\left(E_{i n j} \approx 56 \mathrm{keV}\right)$ at $r=1.13 \mathrm{~m}$ during shot \#29881. For this shot, the spatial and temporal resolutions of the FIDA diagnostics were about $10 \mathrm{~cm}$ and $2 \mathrm{~ms}$, respectively. The expected signals from each view before and after the sawtooth crash are modelled with FIDASIM ${ }^{16}$ based on TRANSP simulations (Fig. 3) in which fast ions are modelled in NUBEAM. ${ }^{17}$ The predicted FIDA spectra and the measurements are plotted in Fig. 1. At low Doppler-shifts from the D-alpha emission line at $656.1 \mathrm{~nm}$, the FIDA signal is masked by the much stronger thermal D-alpha and beam emissions. This is illustrated by shaded areas in Fig. 1. Therefore only the wings of the FIDA spectra are accessible. For the toroidal and vertical views, FIDA measurements are accessible in the ranges between [660.2-662.25] $\mathrm{nm}$ and [650.96-654.5] nm, respectively.

The measured signal in a given wavelength range relates to regions in velocity-space through the so-called fast-ion weight 

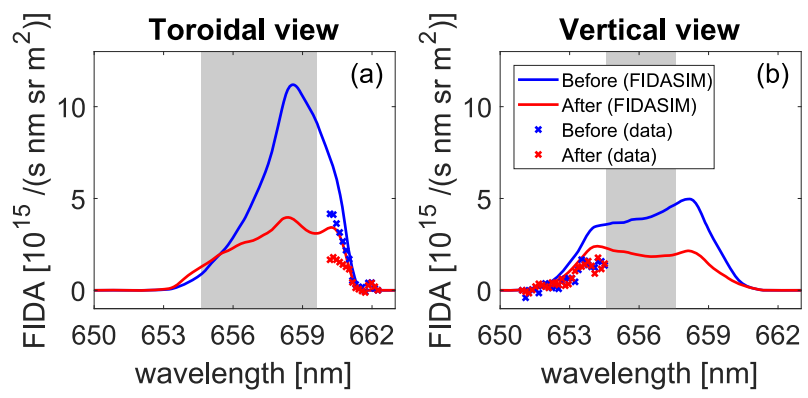

FIG. 1. Central measurements and FIDASIM simulations of the toroidal (a) and vertical (b) views before and after the sawtooth crash. The shaded areas mark the regions where the beam or thermal-ion emissions dominate the FIDA signal.

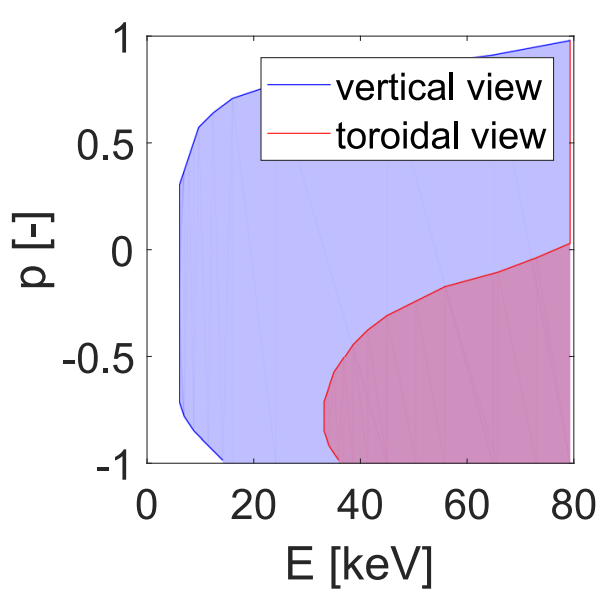

FIG. 2. $(E, p)$-space interrogation regions related to the experimentally accessible wavelength ranges in the toroidal $([660.2-662.25] \mathrm{nm})$ and vertical ([650.96-654.5] $\mathrm{nm})$ views.

functions. ${ }^{18}$ The weight function depends on the observed wavelength range and the viewing angle $\phi$ between the line-ofsight and the local magnetic field in the measurement volume. Hence the toroidal $\left(\phi_{t}=36^{\circ}\right)$ and vertical $\left(\phi_{v}=104^{\circ}\right)$ views are sensitive to different regions in velocity-space. The shaded areas in Fig. 2 depict the total interrogation regions related to the experimentally accessible wavelength domains for the toroidal and vertical views. These are shown in the widely used energy $E=\frac{1}{2} m_{D} v^{2}$ and pitch $p=v_{\|} / v$ space where $v$ is the fast-ion speed and $v_{\|}$is its velocity component along the magnetic field. From this, it is evident that the accessible part of the spectra from the toroidal view is sensitive to high energies $(\sim 40-80 \mathrm{keV})$ at negative pitches, whilst observations from the vertical view are sensitive to much lower energies in a broader pitch-range. Since the beam injection direction in MAST is counter to the toroidal field, most of the beam ions have negative pitches.

\section{TOMOGRAPHIC INVERSION METHODS USING PRIOR INFORMATION}

Fast-ion velocity-space tomography is based on finding the solution $F^{*}$ to the mathematically ill-posed problem

$$
W F^{*}=S \text {, }
$$

where $W$ is the transfer matrix containing the weight functions and $S$ is the measured signal. ${ }^{19-21}$ In order to find a stable solution, first-order Tikhonov regularization ${ }^{22}$ has proven a useful tool in tomography studies. ${ }^{7,12,13,23,24}$ Here the solution is regularized by introducing a penalty term that favours solutions with small gradients. The solution $F^{*}$ is then the distribution $F$ that minimizes the sum of the residual norm of the original problem [Eq. (1)] and the norm of the regularization of the solution. This can be written as

$$
F^{*}=\arg \min _{F}\left\|\left(\begin{array}{c}
W \\
\lambda_{1} L_{1}
\end{array}\right) F-\left(\begin{array}{c}
S \\
0
\end{array}\right)\right\|_{2},
$$

where $L_{1}$ is the gradient matrix ${ }^{12}$ and $\lambda_{1}$ is the regularization parameter. Here the indices refer to the order of the Tikhonov regularization. The regularization parameter determines the strength of the regularization and is, in Sec. IV, chosen such that the solution error is minimized.

To improve the solution, additional prior-information can be included in the so-called high-definition tomography. High-definition velocity-space tomographies of fast ions in fusion plasmas have included non-negativity, nullmeasurements, known peak-locations, and numerical simulations ${ }^{13}$ as prior information. Specifically, studies have shown that adding the non-negativity constraint, i.e., $F^{*} \geq 0$, improves the solution. ${ }^{7,13}$ Therefore, this is used throughout this study.

\section{A. Null-measurements as a hard constraint}

Including null-measurements as a hard constraint in the reconstructions sets the solution to zero in specific parts of velocity-space based on spectral observations. Nullmeasurements are the parts of the spectra where the observed light is below the detection threshold of the instrument. ${ }^{13} \mathrm{In}$ Fig. 1, the null-measurements are above $661.48 \mathrm{~nm}$ in the toroidal view and below $651.4 \mathrm{~nm}$ in the vertical view. The associated velocity-space is to the right of the dashed curved lines in Fig. 3, which agrees well with scarcely populated regions in the neoclassical TRANSP simulations both before and after the sawtooth crash. Previously, null-measurements have been implemented by constraining the solution to be

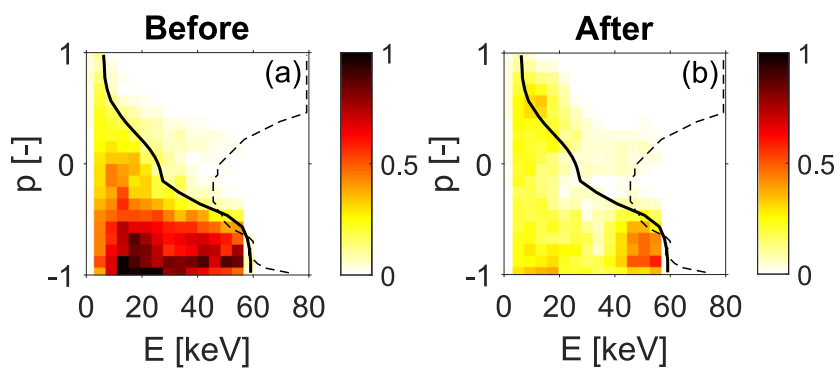

FIG. 3. TRANSP simulations of the fast-ion distributions in $(E, p)$-space at $r=1.13 \mathrm{~m}$, before (a) and after (b) the sawtooth crash. The vertical black line indicates the lower limit of the velocity-space that we seek to reconstruct in Sec. IV. The curved black line is the lower limit of the unlikely velocity-space region defined from the TRANSP simulations. The null-measurement region lies to the right of the dashed line. The same normalisation factor is used in both plots. 
zero at the coordinates $\left(E_{0}, p_{0}\right)$ in the regions covered by null-measurements, i.e., $F\left(E_{0}, p_{0}\right)=0$. This method removes artifacts in the null-measurement region. However, it might introduce artifacts at the sharp boundary between the tomography and null-measurement region. A second concern is that the hard constraint makes it impossible to detect any ions in the null-measurement region. ${ }^{13}$ Our new approach, presented in Sec. III B, overcomes these limitations by changing the hard constraint to a penalty.

\section{B. Unlikely velocity-space defined from null-measurements and neoclassical simulations}

It is only unlikely but not strictly impossible to have an ion in the null-measurement region. Therefore, we propose to penalize the solution with increased penalty strength further into the region instead of forcing the solution to zero. We refer to the region where the presence of ions is unlikely as the unlikely velocity-space. The penalty allows fast ions in this region, but still suppresses clear artifacts. In addition, the smooth nature of the penalty function removes the sharp boundary into the unlikely velocity-space often appearing in tomographies using a hard constraint.

The unlikely velocity-space method is implemented by introducing an additional penalty term in the Tikhonov problem of Eq. (2). This term is designed to suppress solution norms in the unlikely velocity-space without penalizing the solution outside this region. This is encoded in the function $\xi=\xi(E, p)$ that is multiplied onto the zeroth-order Tikhonov penalty operator $L_{0}$ which is the identity matrix. The strength of the penalty is determined by the regularization parameter $\lambda_{0}$ chosen to be as low as possible but still strong enough to suppress clear artifacts in the unlikely velocity-space. This can be determined by inversions of synthetic measurements where the true solution is known such that artifacts can be identified. In order to improve the reconstruction, the solution is still sought to be smooth over the entire domain. Together with the non-negativity constraint, the solution of the unlikely velocity-space method takes the form

$$
F^{*}=\arg \min _{F}\left\|\left(\begin{array}{c}
W \\
\lambda_{1} L_{1} \\
\lambda_{0} \xi L_{0}
\end{array}\right) F-\left(\begin{array}{c}
S \\
0 \\
0
\end{array}\right)\right\|_{2} \text { subject to } F^{*} \geq 0 .
$$

We use this method on both the null-measurement region and in the region where the TRANSP simulations suggest marginal fast-ion densities below the detection limit of the diagnostic. For NBI populations, there is usually a fairly sharp transition between densely and scarcely populated velocity-space regions. Hence, these regions can be distinguished from each other as shown by the separation lines in Fig. 3. We then assume that the larger the distance from the separation line into the unlikely velocity-space, the lower the likelihood of finding ions. Here the penalty function $\xi$ is chosen to increase exponentially with increased energy from the separation line and takes the forms shown in Fig. 4. The specific shapes of the penalty functions are chosen such that the solution errors of the optimal reconstructions from signals created from the TRANSP simulations are minimized. For consistency, these values are also used in the reconstructions of the

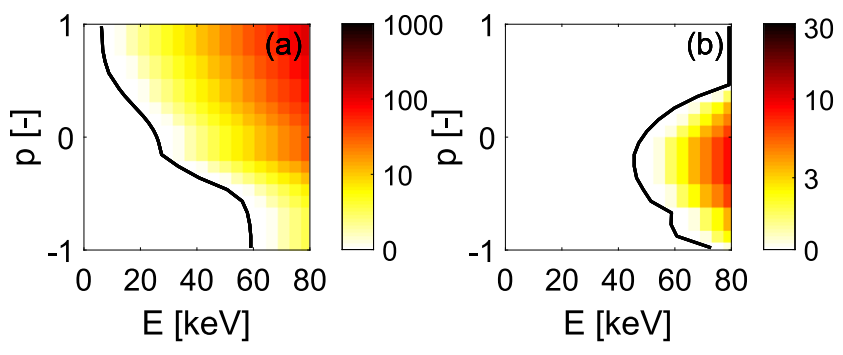

FIG. 4. Exponentially growing penalty function $\xi$ above what is neoclassically expected by the TRANSP simulations (a) and in the null-measurement region (b).

distributions from the FIDASIM and measurement signals in Sec. V.

Even though this method does not force the solution to zero in the unlikely velocity-space, the solution will be more strongly suppressed in this region. Therefore, this method must be used with care on real experimental data where the true solution is not known, and features removed from the less-constrained solutions by this method must be deemed artifacts before being suppressed. This is especially true when assuming only a low likelihood of ions above the TRANSP separation line, where the imposed unlikely velocity-space is not deduced from measurements but rather from neoclassical simulations. This method assumes that TRANSP correctly computes the regions with marginal fast-ion densities, but makes no assumption about the regions with significant fastion densities. Nevertheless, the purpose of this study is not to verify the simulation shape from experimental measurements. Instead we wish to improve the tomographic reconstructions of fast-ion distributions from two-view FIDA measurements in MAST where the non-negativity and hard null-measurement constraints turn out to be insufficient.

\section{INVERSIONS OF SYNTHETIC SIGNAL}

In order to determine the effect of the presented inversion methods on the scarce two-view FIDA signals, the fastion distribution is reconstructed from a synthetic signal with added noise generated from the TRANSP simulation of the distribution before the sawtooth crash [Fig. 3(a)].

Figure 5 shows the resulting reconstructions. Panels (a) and (b) show high-definition inversions as applied in previous studies. ${ }^{13,23}$ Panels (c) and (d) show high-definition inversions using our new method where we identify the unlikely velocity-space before the inversions and solve the problem according to Eq. (3). The distribution in panel (c) is inferred from the unlikely velocity-space method for the region above the neoclassically expected limit, whilst panel (d) gives the solution from the unlikely velocity-space method on the null-measurement region as an alternative to the hard null-measurement constraint. Using only the non-negativity constraint [Fig. 5(a)], the solution is plagued by high densities within two parabola-shaped bands at energies above what is neoclassically expected. Since here we are reconstructing based on synthetic measurements, we know the true solution [Fig. 3(a)]. Hence, we know that these bands are artifacts. These artificial bands follow the outer edge of the 

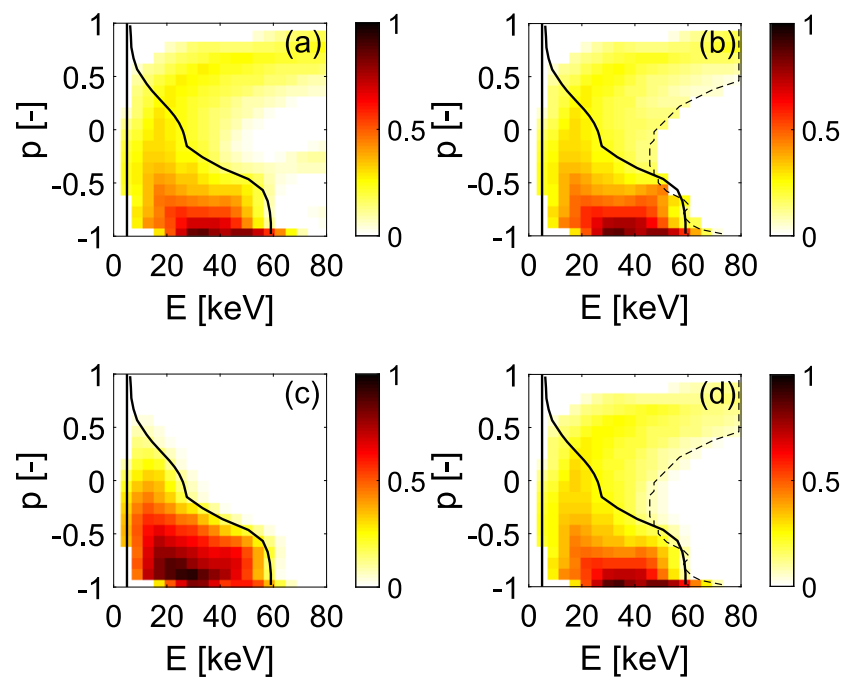

FIG. 5. Inversions of the synthetic signal generated from the TRANSP simulation of the distribution before the sawtooth crash. Each panel uses different prior information: (a) non-negativity, (b) non-negativity and nullmeasurement constraint, (c) the unlikely velocity-space method above what is neoclassically expected, and (d) the unlikely velocity-space method in the nullmeasurement region, with $\lambda_{1}=5 \times 10^{9}$ and $\lambda_{0}=1$. All plots are normalized by the same factor as in Fig. 3 .

interrogation regions of the two views (see Fig. 2). Including the hard null-measurement constraint in the reconstruction [Fig. 5(b)] improves the solution for negative pitches, where the null-measurement region nearly limits the solution to the upper boundary of the TRANSP simulation. However, since only the redshifted part of the toroidal view is accessible, no null-measurements are available to prove that the positivepitch band is an artifact. In addition, the null-measurement constraint introduces a sharp boundary between the nullmeasurement region and the available velocity-space. This sharp boundary is replaced by a smoother decay when replacing the hard null-measurement constraint [Fig. 5(b)] with the exponentially growing penalty term in the null-measurement region in panel (d). The removal of the sharp boundary is most clear near $60 \mathrm{keV}$ at pitches below -0.5 . Hence, substituting the hard null-measurement constraint with the unlikely velocity-space method in the null-measurement region will likely prove favourable in tomography problems in the future. However, here this method does not remove the prominent artificial band at positive pitches. Using instead the unlikely velocity-space method derived from the TRANSP simulation, the artificial band at positive pitches is additionally suppressed and the solution is strongly improved over the entire domain. The suppression of densities in the unlikely velocity-space additionally leads to elevated densities in the neoclassically expected densely populated region of velocity-space which improves the match with TRANSP also in this region.

We conclude that the unlikely velocity-space method assuming low probabilities of fast-ion densities above what is neoclassically expected overcomes some of the problems encountered in previously suggested methods for fast-ion tomography from scarce datasets. This enables the study of the impact of a sawtooth crash on the fast-ion velocity distribution reconstructed from the dual-view FIDA diagnostic at MAST in Sec. V.

\section{THE FAST-ION DISTRIBUTION BEFORE AND AFTER A SAWTOOTH CRASH}

Here we study the effect of the sawtooth crash on the fast-ion density using both the FIDASIM simulations and the measured signals presented in Fig. 1. The fast-ion densities are obtained by integration of the velocity distribution functions. ${ }^{11}$ The measurements lie significantly below the FIDASIM simulations (see Fig. 1) which is probably in part explained by a calibration error. Therefore, we make no statement about the absolute fast-ion densities, but focus on the relative sawtooth crash size, which is not affected by the scaling as it is the same before and after the crash. Note, however, that in the vertical view, the difference between the FIDASIM signal and the measurements is larger before than after the sawtooth crash. This is discussed later in this section.

We focus on the fastest ions above $30 \mathrm{keV}$ as the toroidal view does not cover lower energies. Figure 6 shows the reconstructions of the fast-ion distributions from the noisy FIDASIM simulations [panels (a) and (b)] and the reconstructions from measurements [panels (c) and (d)] before and after the sawtooth crash. Here we use the unlikely velocity-space method assuming only a small likelihood of ions possessing energies and pitches above the expected limit in the neoclassical TRANSP simulations. The inversion shows a marginally longer tail above the injection energy than the TRANSP simulation. It is not possible to tell from the data if this corresponds to higher fast-ion densities in the experiment or if it is an artifact.

In the reconstructions, the generally lower fast-ion densities from the measurements compared to the densities obtained from the FIDASIM reconstructions are explained by the lower radiance detected in the experimental signals compared to what was expected by FIDASIM, which is probably related to the
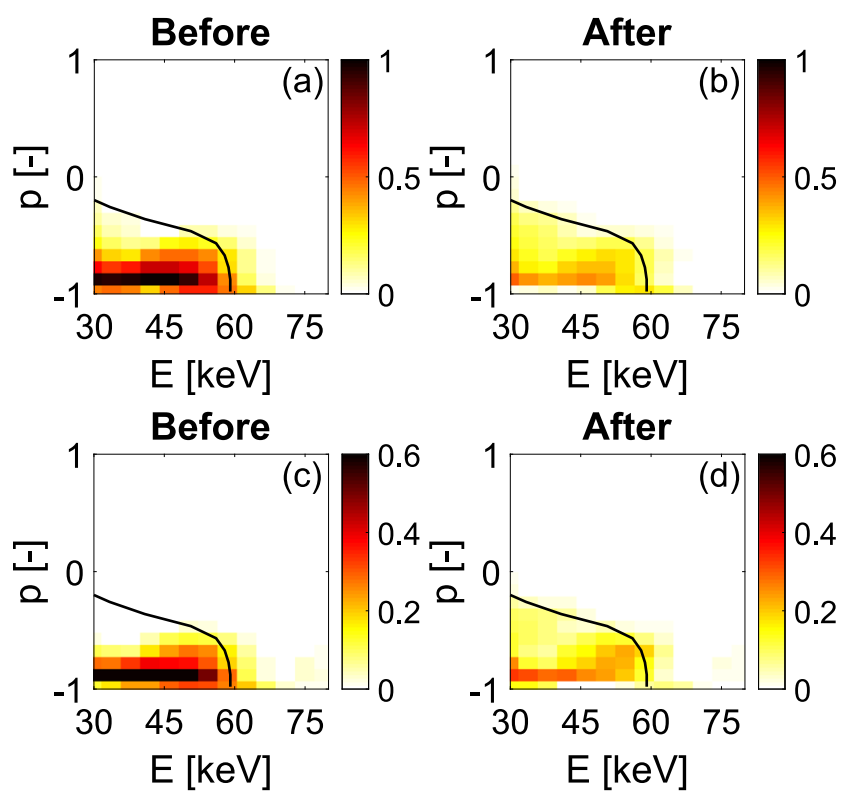

FIG. 6. Reconstructions of the FIDASIM [(a) and (b)] and experimental [(c) and (d)] signals before and after the sawtooth crash using $\lambda_{1}=5 \times 10^{9}$ and $\lambda_{0}=1$. All plots are normalized by the same factor as in Fig. 3. Note that the upper color scale limit in panels (a) and (b) differs from the one in panels (c) and (d). 
uncertain calibration mentioned above. For energies above $30 \mathrm{keV}$, the TRANSP simulation predicts a fast-ion density decrease of $48 \%$ due to the sawtooth crash. In the reconstructions from the FIDASIM and experimental signals, the corresponding fast-ion density decreases are $46 \%$ and $42 \%$, respectively. At ASDEX Upgrade, the measured decrease of the fast-ion density by velocity-space tomography was also smaller than in a corresponding TRANSP simulation. ${ }^{13}$

The slightly smaller relative fast-ion density decrease in the reconstructions from the measurements, compared to the density decrease obtained from the FIDASIM signals, originates partly from the vertical view measurements that stay approximately constant over the sawtooth crash. This agrees with observations from other machines where sawtooth crashes most strongly affect passing fast ions. ${ }^{12,25,26}$ In ASDEX Upgrade, this pitch-dependence of the redistribution of energetic ions due to sawtooth crashes has recently been modelled with good agreement between the measurements and model, ${ }^{27}$ and future tomographic studies of sawtooth crashes might give further evidence on whether this is a consistent feature in tokamaks.

\section{CONCLUSION}

We studied velocity-space tomography based on the dualview FIDA measurements at MAST which constitutes a scarce-data problem. For this type of scarce-data problem, the use of prior information is essential to obtain usable inversions. However, reconstructions of the fast-ion velocity distributions using only previously introduced tomographic methods are plagued by clearly artificial increased-density structures at positive pitches covered by only the vertical view. To overcome this, we introduce a new way of including prior information. We identify the portion of velocity-space that is unlikely to have significant fast-ion densities. We then impose an exponentially growing penalty function in the unlikely velocityspace. Ideally, this region should be determined solely by null-measurements, which proves to be an advantageous alternative to the hard null-measurement constraint that has previously been used in high-definition tomography. However, in this study, the null-measurement penalty method does not suppress all clear artifacts. We therefore propose to use the neoclassical TRANSP simulations to determine the unlikely velocity-space by assuming it unlikely that ions possess energies and pitches far above what is neoclassically expected. This additional prior information results in a strongly improved solution. Using this method, the fast-ion velocity distributions before and after a sawtooth crash have been reconstructed from experimental measurements. Over the sawtooth crash, the relative decrease in the fast-ion density for energies above $30 \mathrm{keV}$ is marginally smaller than in the TRANSP simulations as was also found at ASDEX Upgrade.

\section{ACKNOWLEDGMENTS}

We thank the ITPA Energetic Particle Physics Topical Group for its support. This work has been carried out within the framework of the EUROfusion Consortium and has received funding from the Euratom research and training programme 20142018 under Grant Agreement No. 633053 and from the RCUK Energy Programme (Grant No. EP/P012450/1). The views and opinions expressed herein do not necessarily reflect those of the European Commission.

${ }^{1}$ S. D. Pinches et al., Plasma Phys. Controlled Fusion 46, B187 (2004).

${ }^{2}$ W. W. Heidbrink, Rev. Sci. Instrum. 81, 10 D727 (2010).

${ }^{3}$ W. W. Heidbrink et al., Plasma Phys. Controlled Fusion 46, 1855 (2004).

${ }^{4}$ J. Huang et al., Rev. Sci. Instrum. 87, 11E542 (2016).

${ }^{5}$ T. Ito et al., Rev. Sci. Instrum. 81, 10D327 (2010).

${ }^{6}$ M. Podestá et al., Rev. Sci. Instrum. 79, 10E521 (2008).

${ }^{7}$ M. Weiland et al., Plasma Phys. Controlled Fusion 58, 025012 (2016).

${ }^{8}$ B. Geiger et al., Plasma Phys. Controlled Fusion 59, 115002 (2017).

${ }^{9}$ C. A. Michael et al., Plasma Phys. Controlled Fusion 55, 095007 (2013).

${ }^{10}$ M. Cecconello et al., Plasma Phys. Controlled Fusion 57, 014006 (2015).

${ }^{11}$ B. Geiger et al., Nucl. Fusion 55, 083001 (2015).

${ }^{12}$ A. S. Jacobsen et al., Plasma Phys. Controlled Fusion 58, 045016 (2016).

${ }^{13}$ M. Salewski et al., Nucl. Fusion 56, 106024 (2016).

${ }^{14}$ B. C. Stratton et al., Nucl. Fusion 36, 1586 (1996).

${ }^{15}$ A. Kirk et al., Nucl. Fusion 57, 102007 (2017).

${ }^{16}$ W. W. Heidbrink et al., Commun. Comput. Phys. 10, 716741 (2011).

${ }^{17}$ A. Pankin et al., Comput. Phys. Commun. 159, 157 (2004).

${ }^{18}$ M. Salewski et al., Plasma Phys. Controlled Fusion 56, 105005 (2014).

${ }^{19}$ M. Salewski et al., Nucl. Fusion 52, 103008 (2012).

${ }^{20}$ M. Salewski et al., Nucl. Fusion 53, 063019 (2013).

${ }^{21}$ M. Salewski et al., Nucl. Fusion 54, 023005 (2014).

${ }^{22}$ P. C. Hansen, Discrete Inverse Problems: Insight and Algorithms (SIAM, 2010).

${ }^{23}$ M. Salewski et al., Nucl. Fusion 57, 056001 (2017).

${ }^{24}$ M. Weiland et al., Nucl. Fusion 57, 116058 (2017).

${ }^{25}$ C. M. Muscatello et al., Plasma Phys. Controlled Fusion 54, 025006 (2012).

${ }^{26}$ S. K. Nielsen et al., Plasma Phys. Controlled Fusion 52, 092001 (2010).

${ }^{27}$ F. Jaulmes et al., Nucl. Fusion 56, 112012 (2016). 